\title{
THE ROTATION OF THE MAIN-SEQUENCE COMPONENTS OF ALGOL-TYPE SEMI-DETACHED ECLIPSING BINARIES*
}

\author{
E. P. J.VAN DEN HEUVEL ${ }^{\dagger}$
}

Lick Observatory, University of California, Santa Cruz, Calif., U.S.A.

\begin{abstract}
Newly determined rotational velocities of the main-sequence components of 14 Algol-type semi-detached systems and of 2 detached systems are presented. Combination of these data with the existing data on the rotation of the components of semi-detached systems shows that (i) in systems with primaries of spectral type $\mathrm{B} 8$ or later and with $P<5$ days, deviations from synchronism between rotation and revolution are small in 14 out of 15 cases. The average rotational velocity of the primaries in such systems is $75 \mathrm{~km} / \mathrm{sec}$, viz. only $40 \%$ of the average rotational velocity of single mainsequence stars in the same spectral region: (ii) primaries of spectral type earlier than B8 in systems with short as well as long periods tend to rotate more than twice as fast as one would expect from synchronism. A tentative explanation for these results is presented.
\end{abstract}

In close binaries with periods less than about 4 days and with both components on the main sequence the axial rotation of the components is generally observed to be synchronised with the orbital revolution (Plaut, 1959; Olson, 1968). However, in semi-detached Algol-type systems with periods of less than 4 days such a synchronism is not always observed; for instance in the systems of U Cephei (Struve, 1944, 1963) and RZ Scuti (Hansen and McNamara, 1959) the main-sequence components rotate much faster than would be expected in case of synchronism.

Algol-type semi-detached (s-d) systems are expected to be the products of extensive mass exchange in evolving close binaries. During such a mass exchange both the binary period and the rotational angular momentum of the components may change. In the computations of the evolution of close binaries one generally assumes for computational reasons that no exchange between orbital angular momentum of the exchanged matter and rotational angular momentum of the components occurs. However, the above mentioned observations of non-synchronism, as well as computations of particle trajectories in close binary systems (cf. Kruszewski, 1966) show that such an exchange of angular momentum probably takes place in nature. One therefore does not a priori expect the components of s-d systems to rotate synchronously with the orbital motion, especially not during or just after the stage of mass exchange. One may then wonder whether the deviations from synchronism will last for a long time after the mass exchange is terminated, or whether tidal (re-)synchronisation will occur in a fairly short period of time. One may try to answer such a question by examining the rotation of primaries of a random sample of s-d systems, and see how large the percentage of synchronous rotators is. Since the random sample probably will show a random distribution of ages (following the mass exchange stage) the fraction of synchronised primaries in the random sample is then expected to be equal

* Contributions from the Lick Observatory, No. 305.

† On leave of absence from Sterrewacht Sonnenborgh, Utrecht, The Netherlands, July 1, 1968 September 1, 1969. 


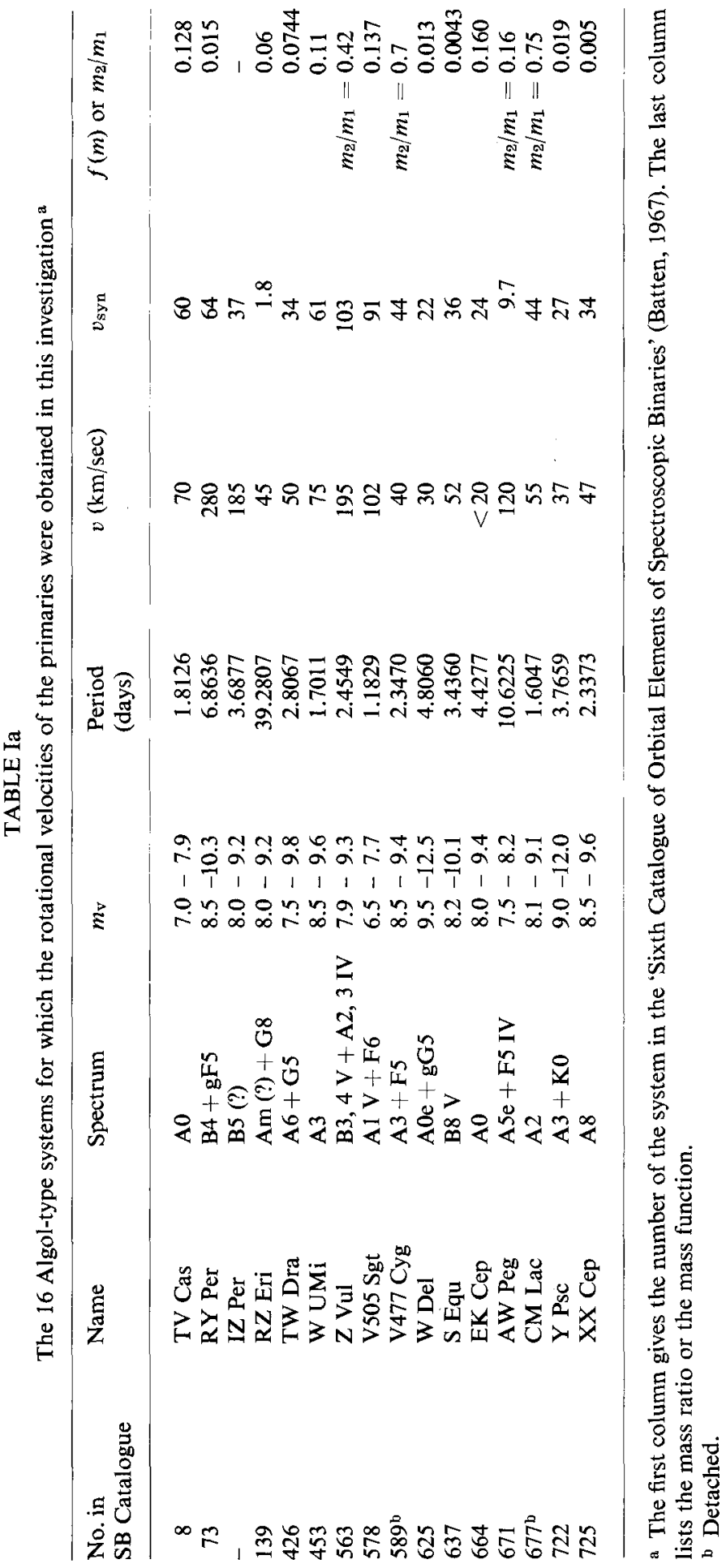




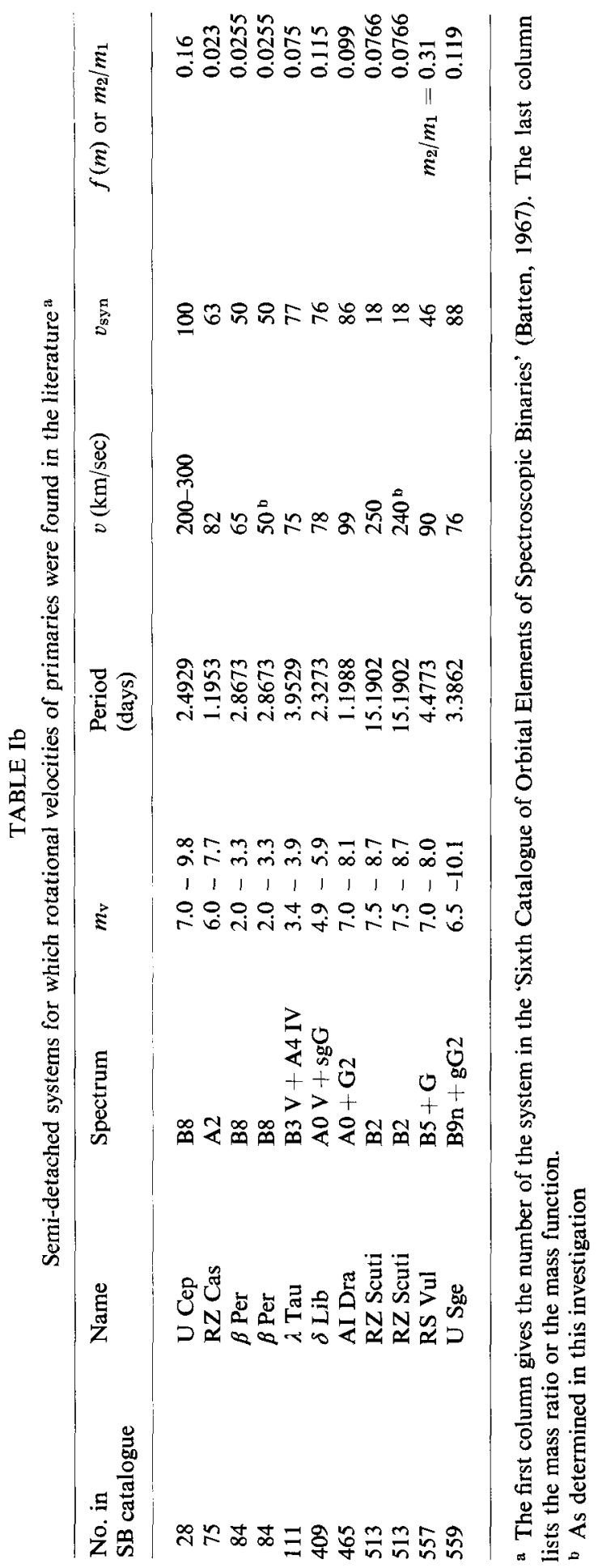


to the fraction of the lifetime of an Algol-type s-d system (after the mass exchange stage) during which its primary rotates synchronously.

Inspection of the literature showed us that for only nine s-d systems are accurate rotational velocities, determined from spectra with dispersions of about $20 \AA / \mathrm{mm}$ or better, available. The data for these systems are given in Table Ib. The rotation of Algol was determined by Slettebak (1954); the values for U Cephei and RZ Scuti were taken from the above mentioned sources: the data for the six other stars were given by Olson (1968). Since this sample is rather small it was decided to study the rotation of the 16 systems in Table Ia. Apart from the systems already studied by others, these are practically all Algol-type systems in the 'Sixth Catalogue of Orbital Elements of Spectroscopic Binaries' (Batten, 1967) brighter than 9..5 at maximum and accessible at Lick Observatory during the late spring and early summer. It was later found that the systems of CM Lac and V477 Cyg are not s-d, hence 14 s-d systems in our sample remain.

Of each system, as well as of Algol and RZ Scuti, at least two spectra were obtained. We used the $20^{\prime \prime}$ Schmidt camera of the coudé spectrograph of the $120^{\prime \prime}$ telescope, with a dispersion of $16 \AA / \mathrm{mm}$ at $4300 \AA$. The widening used in general was $0.5 \mathrm{~mm}$, although some plates were taken with $0.35 \mathrm{~mm}$ widening. In addition spectra of 20 bright A and B stars with known rotational velocities (Slettebak, 1949, 1954, 1955) were taken, which served as standards. All spectra were taken on baked Kodak Ila-O plates. Direct intensity tracing was made of all plates. For the determinations of the rotational velocities the line $\mathrm{Mg}_{\text {II }} 4481 \AA$ was used for spectral types later than B8. For spectral type B 8 and earlier the line He I $4026 \AA$ was used. For spectral type B8 both lines were used. The rotational velocities were determined in a way somewhat similar to the one used by Abt and Jewsbury (1969), viz. curves of $v \sin i$ vs. halfwidth and central depth of the standard lines were constructed for the standard stars. The calibration of the halfwidth of $\mathrm{Mg}$ II 4481 against Slettebak's rotational velocities indicates a p.e. per star of about $10 \%$ in $v \sin i$. The central depth gives a less well defined relation, with a p.e. about twice as large. Measurements of the halfwidth and central depth of the standard lines in the spectra of the Algol-type systems then give 2 values of $v \sin i$. Thirdly, direct visual comparisons were made between the line profiles for the standard stars and the program stars, from which another value of $v \sin i$ was obtained. A similar procedure was followed with respect to the He 4026 line. Here the p.e. of the $v \sin i$ values derived from the halfwidth relation is about $15 \%$, and of the central depth relation about $20 \%$.

The finally adopted rotational velocity for each star is the average of the 6 values obtained from the 2 plates per star; in determining this average, the values obtained from the central depth were given half weight only.

From the scatter in the 6 values of $v \sin i$ for each star we estimate the internal p.e. in the final average to be about $\pm 10 \%$. Table $I$ lists the finally adopted values of the rotational velocity $v$; the inclinations $i$ were taken from the best available orbital studies. The table furthermore lists the velocity $v_{\text {syn }}$ expected in the case of synchronism. For the computation of this velocity we used the best available determination 


\section{TABLE II}

The spectral type vs. radius relation adopted for systems for which no accurate radius determinations were available

\begin{tabular}{ll}
\hline Spectral type & Radius (in $R_{\odot}$ ' \\
\hline A5 & 1.87 \\
A3 & 1.92 \\
A0 & 2.00 \\
B8 & 2.50 \\
B5-6 & 3.3 \\
B4-5 & 4.0 \\
B3 & 4.7 \\
B2 & 5.2 \\
\hline
\end{tabular}

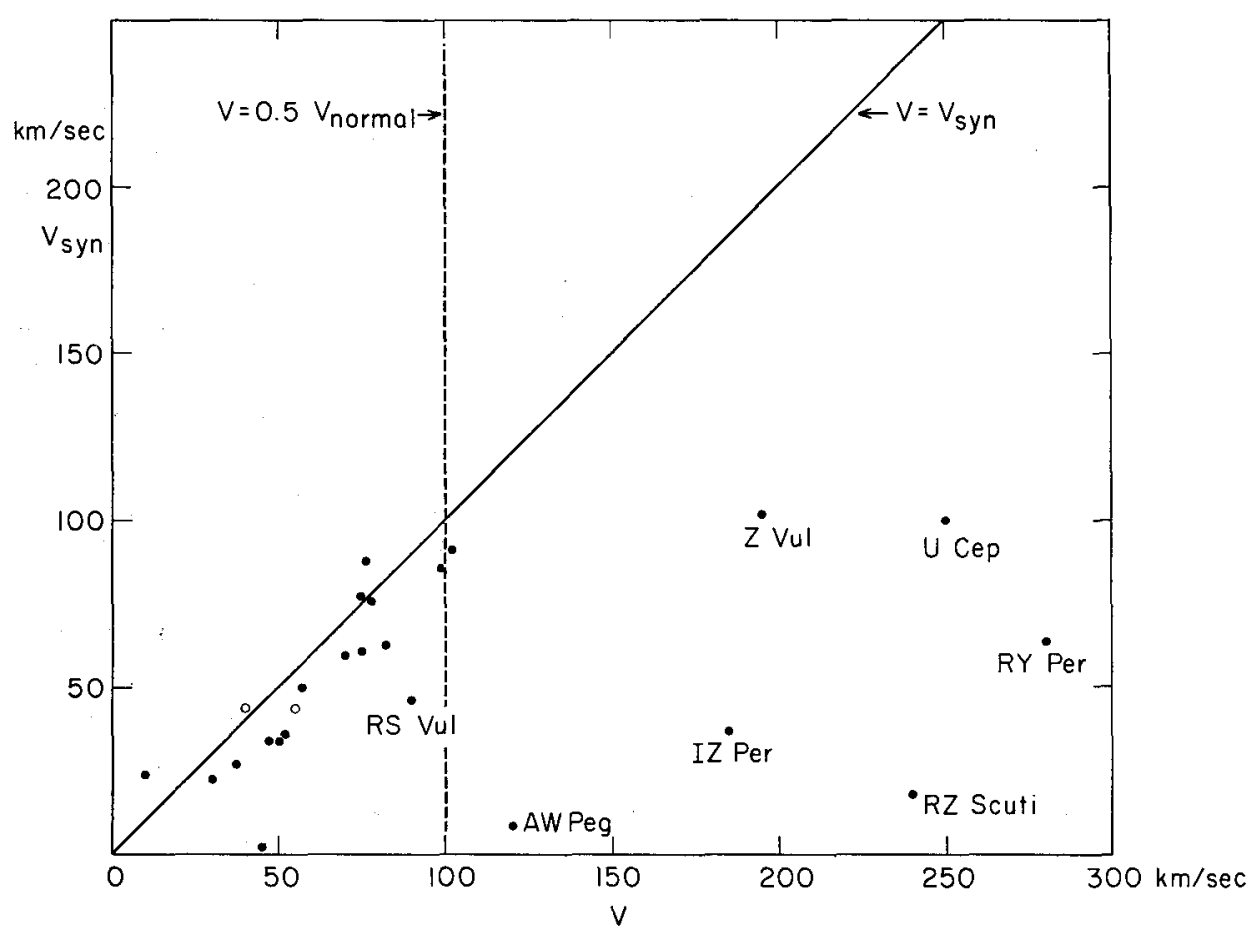

Fig. 1. Plot of the rotational velocities $v$ of primaries of the Algol-type systems from Table I against the velocities $v_{\text {syn }}$ expected in case of synchronism between rotation and revolution. Filled circles represent semi-detached systems; open circles indicate the 2 detached systems.

of the radius from photometric and spectroscopic investigations. In cases in which no value for the radius was available we used the main-sequence relation for radius vs. spectral type given by Harris et al. (1963) as specified in Table II.

In Figure $1 v$ is plotted vs. $v_{\text {syn }}$. The non-semi-detached systems are indicated by open circles. The figure and the table show that within the observational uncertainties, 
in about $67 \%$ of all cases rotation and revolution in s-d systems are synchronised. There seems to be a slight tendency to rotate about $10 \mathrm{~km} / \mathrm{sec}$ faster than synchronous, although it is not certain whether this is a real effect. It is interesting to notice that all non-synchronous primaries in systems with $P<5$ days have spectral type B8 or earlier. Systems with $P<5$ days and primaries of spectral type $B 8$ or later show synchronous rotation in 14 out of 15 cases. The systems RZ Eri and AW Peg, which both have $P>10$ days, have primaries which still rotate much more slowly than single A-or B-type main-sequence stars. The table shows that the mean rotational velocity of primaries of type B8 or later in s-d systems is about $75 \mathrm{~km} / \mathrm{sec}$, which is about $40 \%$ of the average value for single late $B$ and early A stars.

Contrary to what is observed for spectral type B8 and later, the primaries of type earlier than $\mathrm{B} 8$ rotate on the average more than twice as fast as expected in case of synchronism. $\lambda$ Tauri is the only synchronous rotator in this group of 6 .

These results seem to indicate that in systems with primaries of spectral type B8 or later, and with periods less than 5 days, synchronism is restored quite rapidly after the mass exchange stage, whereas in systems with earlier type primaries resynchronisation might take more than three quarters of the lifetime of the system, after the stage of mass exchange.

The interpretation of this difference in rotational behaviour may perhaps be found in the way in which these systems were produced. For the types of evolution of close binary systems we will use here the notation by Kippenhahn and Weigert (1967), viz.: case $\mathrm{A}$ for evolution in which the primary already fills its Roche lobe before the end of core hydrogen burning, case B for the evolution in which the primary fills the Roche lobe after the end of core hydrogen burning but before the onset of helium burning. From the work of Kippenhahn et al. (1967) and of Refsdal and Weigert (1969) it is known that Algol-type s-d systems can be produced either by case B evolution with initial primary mass smaller than about $2.8 M_{\odot}$, or by case A evolution for any value of the initial primary mass. The primary masses of the Algol-type systems produced in case B are not expected to be much larger than $4 M_{\odot}$; for an average initial mass ratio of primary and secondary of 2.0, and an initial primary mass of $2.8 M_{\odot}$, the mass of the new primary after the mass exchange is expected to be about $3.8 M_{\odot}$ (cf. Kippenhahn et al., 1967), which is about the mass of a B8 main-sequence star. For this reason, Algol-type systems produced by evolution according to case $\mathrm{B}$ are expected to have in general only primaries of spectral type B8 or later.

On the other hand, although case A can in principle occur for any spectral type, it is most common among stars earlier than about B8, as Table III shows. The left-hand side of this table shows the distribution of periods of unevolved B-type spectroscopic binaries as given in the Sixth Catalogue (Batten, 1967); the right-hand side of the

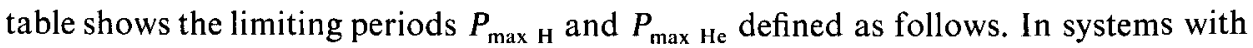
$P<P_{\max H}$ the primary already fills the Roche lobe before the end of core hydrogen burning; in systems with $P<P_{\max H e}$ it fills the Roche lobe before the onset of core helium burning. Consequently, systems with $P<P_{\max H}$ are in case $\mathrm{A}$, and systems with $P_{\max \mathrm{H}} \leqslant P<P_{\max H e}$ are in case B. Comparison of the left- and right-hand side of 
TABLE III

Left: the distribution of periods of unevolved B-type spectroscopic binaries in the 'Sixth Catalogue of Orbital Elements of Spectroscopic Binaries' (Batten 1967). Right: the periods $P_{\max }$ H and $P_{\max }$ He (defined in the text) for three stellar masses, for an original mass ratio of secondary and primary of 0.5 .

\begin{tabular}{lccccc}
\hline$P$ (days) & B0-B5 & B6-B9.5 & $M$ & $P_{\max \text { H }}$ & $P_{\max \text { He }}$ \\
\hline$<1$ & $0.9 \%$ & $2.6 \%$ & & & \\
$1-1.5$ & 7.9 & 7.9 & $3 M_{\odot}(\sim \mathrm{B} 8)$ & $0^{\mathrm{d}} .99$ & $35^{\mathrm{d}} .4$ \\
$1.5-2$ & 9.6 & 7.9 & & & \\
$2-3$ & 21.1 & 15.8 & $5 M_{\odot}(\sim \mathrm{B} 5)$ & $1^{\mathrm{d}} .36$ & $92^{\mathrm{d}} .2$ \\
$3-9$ & 28.9 & 31.6 & & & \\
$9-27$ & 17.6 & 21.0 & $9 M_{\odot}(\sim \mathrm{B} 2)$ & $2^{\mathrm{d}} .00$ & $406^{\mathrm{d}} .4$ \\
$27-81$ & 7.0 & 7.9 & & & \\
$>81$ & 7.0 & 5.3 & & & \\
\hline
\end{tabular}

Table III shows that case A occurs among about $18 \%$ of the early B stars, but among less than $10 \%$ of the later B systems. In view of the decrease of $P_{\max H}$ with stellar mass, it occurs among only a few per cent of the A stars (which have about the same period distribution for spectroscopic binaries as the later B stars). For this reason, case A is quite common among unevolved spectroscopic binaries of spectral type earlier than about B8, but is quite rare at later types. For this reason one expects most of the s-d systems with primaries of spectral type B8 or later to be the products of case B evolution, whereas systems with primaries of spectral type earlier than about B8 are expected to be mostly the products of case A.

Why then do the products of case A evolution rotate much faster than the products of case B evolution? The reason might be that in case B most of the mass-exchange takes place in a relatively short time, and there is in general no long lasting stage of slow but considerable mass exchange (Kippenhahn et al., 1967; Refsdal and Weigert, 1969). In this case, therefore, once the mass exchange is over, tidal forces can start their synchronising work. On the other hand, in case A only a relatively small amount of mass is transferred rapidly, followed by a long-lasting stage of slow mass exchange (Plavec et al., 1968). Hence, in this case the surface layers of the primary always consist of newly accreted material, presumably of high angular momentum, which did not yet have time to adjust itself to synchronism. We suggest that this may be the reason for the different rotational behaviour of systems with primaries of spectral type earlier and later than about $\mathbf{B} 8$.

One may wonder what will happen to the systems with primaries of spectral type later than about B8, after the subgiant component has evolved into a white dwarf. According to the work of Kippenhahn et al. (1967) and of Refsdal and Weigert (1969) the subgiant has a lifetime of only about 15 to $20 \%$ of the remaining lifetime of the primary. One therefore expects this main-sequence primary to have a white dwarf companion during $80 \%$ or more of its lifetime after the mass exchange. From the fact that, after due allowance for inclination effects, some $2.5 \%$ of the A and B stars are 
primaries in Algol-type s-d systems (Van den Heuvel, 1969a) one expects some 10$12.5 \%$ of the A and B stars to have been primaries in $\mathrm{s}-\mathrm{d}$ systems, and to have a white dwarf companion now. The fact that for spectral type later than about B8 the primaries of Algol-type s-d systems are slow rotators indicates then, that one expects the $10-12.5 \%$ of the late $B$ and $A$ stars which have white dwarf companions, to be relatively slow rotators. This might be the explanation for the observed occurrence of an overabundance of slow rotators among the early A stars (Conti, 1965; Deutsch 1967). Similarly, one expects those blue stragglers in galactic clusters that are evolved spectroscopic binaries to have rotational velocities comparable to those of the stars in Table I. Indeed the A-type blue stragglers in M67 studied by Deutsch (1966) have rotational velocities not larger than about $100 \mathrm{~km} / \mathrm{sec}$ and the blue straggler 40 Cancri in Praesepe is a very slow rotator (Conti et al., 1965). (The position of the blue straggler 68 Tauri in the colour magnitude diagram of the Hyades can be explained by ordinary evolution of a single star (Van den Heuvel, 1969b).)

\section{Acknowledgement}

This research has been supported in part by the ONR under Contract Number N00014-69-C-0155.

\section{References}

Abt, H. and Jewsbury, C. P.: 1969, Astrophys. J. 156, 983.

Batten, A.: 1967, 'Sixth Catalogue of Orbital Elements of Spectroscopic Binaries', Publ. Dom. Astrophys. Obs. 13, 8.

Conti, P. S.: 1965, Astrophys. J. 142, 1594.

Conti, P. S., Wallerstein, G., and Wing, R. F.: 1965, Astrophys. J. 142, 999.

Deutsch, A. J.: 1966, Astron. J. 71, 383.

Deutsch, A. J.: 1967, in The Magnetic and Related Stars (ed. by R. C. Cameron), Baltimore, p. 181.

Hansen, K. and McNamara, D. H.: 1959, Astrophys. J. 130, 792.

Harris, D. L., Strand, K. Aa., and Worley, C. E.: 1963, in Stars and Stellar Systems (Chicago) 3, 273.

Kippenhahn, R. and Weigert, A.: 1967, Z. Astrophys. 65, 251.

Kippenhahn, R., Kohl, K., and Weigert, A.: 1967, Z. Astrophys. 66, 58.

Kruszewski, A.: 1966, Advances Astron. Astrophys. 4, 233.

Olson, E. C.: 1968, Publ. Astron. Soc. Pacific 80, 185.

Plaut, L.: 1959, Publ. Astron. Soc. Pacific. 71, 167.

Plavec, M.: 1968, Advances Astron. Astrophys. 6, 201.

Plavec, M., Kriz, S., Harmanec, P., and Horn, J. 1968, Bull. Astron. Inst. Czech. 19, 24.

Refsdal, S. and Weigert, A.: 1969, Astron. Astrophys. 1, 167.

Slettebak, A.: 1949, Astrophys. J. 110, 498.

Slettebak, A.: 1954, Astrophys. J. 119, 146.

Slettebak, A.: 1955, Astrophys. J. 121, 653.

Struve, O.: 1944, Astrophys. J. 99, 222.

Struve, O.: 1963, Publ. Astron. Soc. Pacific 75, 107.

Van den Heuvel, E. P. J.: 1969a, Astron. J. 74, 1095.

Van den Heuvel, E. P. J.: 1969b, Publ. Astron. Soc. Pacific 81, 815. 


\section{Discussion}

$A b t$ : What periods would you expect for the evolved binaries with white dwarf companions?

Van den Heuvel: About the same as or slightly larger than those of Algol-type semi-detached systems. There is a selection effect which favours detection of Algol-systems with short periods. Due to this effect the systems with a white dwarf component may well have larger average periods than the average period of the Algol-type systems in Table I. The expected percentage of the remnants of Algol-type systems given here is therefore also probably an underestimate.

I would further like to stress that the mass ratios of the systems with white dwarfs are very small, and the binary character of such systems may easily escape detection. 